\title{
FASE PRELIMINAR EN EL PROCESO DE INNOVACIÓN SOCIAL CON LOS PRODUCTORES AGRÍCOLAS Y DE LECHE DEL MUNICIPIO DE VIRACACHÁ - BOYACÁ ${ }^{1}$
}

\section{Yineth Ochoa Rodríguez ${ }^{2}$}

Para citar este artículo: Ochoa, Y. (2015). "Fase preliminar en el proceso de innovación social con los productores agrícolas y de leche del municipio de Viracachá - Boyacá”. Inquietud Empresarial. Vol. XV (1), pp. 53-74

Fecha de recepción: 27 de octubre de 2014 Fecha de aceptación: 30 de enero de 2014

\footnotetext{
1 Artículo de Investigación Científica y Tecnológica elaborado bajo la modalidad de Proyecto de Investigación registrado en la Dirección de Investigación de la Universidad Pedagógica y Tecnológica de Colombia.

2 Estudiante de Administración de Empresas, integrante del semillero de investigación del Grupo de Investigación IDEAS adscrito a la Escuela de Administración de Empresas de la UPTC, Tunja. yinesita88@hotmail.com
} 


\title{
RESUMEN
}

Este proyecto, aborda el desarrollo de la etapa preliminar en un proceso de innovación social, consistente en construir un perfil sociocultural y económico de la comunidad involucrada con la producción agrícola y de leche en el municipio de Viracachá, y de acuerdo con ello se desarrolla un proceso de apoyo, acompañamiento y asesoría a dichos productores para construir un capital social que les ha permitido concertar una estrategia organizacional y un modelo de negocio que articula esta actividad productiva local con cadenas de valor nacionales.

En este manuscrito se identifican los liderazgos existentes en el grupo de productores y las experiencias asociativas vividas. Se establecen necesidades en términos de formación para el trabajo asociativo y para la gestión de organizaciones rurales.

\section{PALABRAS CLAVE}

Innovación social, asociatividad, liderazgo, productores agrícolas, productores de leche.

\section{Preliminary phase in the process of social innovation with agricultural and milk producers of Viracachá - Boyacá}

\begin{abstract}
This project addresses the development of the preliminary stage in a process of social innovation was to build a socio-cultural and economic profile of the community involved with agricultural production and milk in the town of Viracachá, and accordingly is developing a process support, assistance and advice to those producers to build social capital that has allowed them to make an organizational strategy and a business model that articulates this local productive activity with national value chains.

This paper identifies existing leadership in the group of experienced producers and associative experiences. Needs are in terms of training for partnership working and the management of rural organizations.
\end{abstract}

\section{KEY WORDS}

Social innovation, partnership, leadership, farmers, milk producers. 
FASE PRELIMINAR EN EL PROCESO DE INNOVACIÓN SOCIAL CON LOS PRODUCTORES AGRICOLAS Y DE LECHE DEL MUNICIPIO DE VIRACACHA - BOYACÁ

\section{Fase preliminar no processo de inovação social com os produtores agrícolas e de leite do municipio de Viracachá - Boyacá}

\section{RESUMO}

Este projeto, aborda o desenvolvimento da etapa preliminar no proceso de inovação social, consiste em construir um perfil sociocultural e econômico da comunidade envolvida com a produção agrícola e de leite no municipio de Viracahá, e de acordo com ele se desenvolve um proceso de apoio, acompanhamento e assessoria a certos produtores para construir um capital social que lhes permitam concertar uma esratégia organizacional e um modelo de negócio que articula esta atividade produtiva local com cadeias de valor nacionais.

Neste manuscrito se identificam as lideranças existentes no grupo de produtores e as experiências associativas vividas. Se estabelecem necessidades em términos de formação para o trabalho associativo e para a gestão de organizações rurais.

\section{PALAVRAS CHAVE}

Inovação social, associatividade, liderança, produtores agrícolas, produtores de leite.

\section{INTRODUCCION}

La Comisión Económica para América Latina y el Caribe (2011), define la innovación social como "nuevos procesos, prácticas, métodos o sistemas para llevar a cabo procesos tradicionales o tareas nuevas que se hacen con participación de la comunidad y los beneficiarios. Estos se transforman en actores de su propio desarrollo, fortaleciendo así el sentimiento de ciudadanía y mejorando sus condiciones sociales y de vida"; es por esto que en el presente documento se encuentran las pautas para llevar a cabo un proceso de innovación social en los productores agrícolas y de leche en el municipio de Viracachá- Boyacá, esto a petición de dichas personas que en el afán de conseguir protección para su diario vivir desean una cooperación entre el estado, universidad y sector productivo para lograr un proceso donde el beneficio no sea para algunos sino para la comunidad en general (Revista Semana, 2013).

Sin embargo, se observa que este tipo de proyectos son necesarios para 
la subsistencia de los pequeños productores agrícolas y de leche, ya que como se han presentado en días pasados el sector agrícola y de leche, pasa por momentos de gran perdida y recesión debido a las malas decisiones del gobierno en cuanto a importaciones se refiere, decisiones que han afectado al sector agrario en general.

Es en este punto donde la universidad entra a jugar un papel importante dentro de la sociedad, ya que aquí es donde se guarda la esperanza de intervenir ante el gobierno como un ente de conocimiento y sabiduría, para así ayudar a aquellas personas que necesitan con rapidez una solución definitiva a sus problemas y por qué no empezar por diseñar procesos de innovación social que en un principio se centre en los productores agrícolas y de leche en el municipio de Viracachá -Boyacá y en un futuro se extienda a muchos más municipios y sectores productivos que requieran de la asesoría e implementación de dicho proceso.

El propósito del presente trabajo fue el de desarrollar la etapa preliminar del proceso de innovación social con los productores agrícolas y de leche en el municipio de Viracachá - Boyacá; específicamente se buscó:

- Elaborar un perfil socio-cultural y económico de la comunidad productora agrícola y de leche en el municipio en mención.

- Identificar liderazgos y experiencias de trabajo asociativo en la comunidad objeto de estudio

- Formar en los aspectos básicos para el trabajo asociativo y para la gestión de organizaciones asociativas rurales, a los productores agrícolas y de leche en el municipio de Viracacha - Boyacá

\section{PROBLEMA}

- Descripción del problema

El departamento de Boyacá, se ha caracterizado por ser uno de los principales productores del tubérculo de la papa a nivel nacional, con una representación del $90 \%$ de la producción junto con los departamentos de Cundinamarca, Nariño y Antioquía. En esa medida, Boyacá y Cundinamarca, abarcan el $60 \%$ de dicha producción, lo cual evidencia que estos departamentos concentran su economía en el sector agrícola específicamente en el cultivo de la papa. Así, ésta se ha convertido en uno 
de los productos más apetecidos de la canasta familiar teniendo en cuenta que es asequible por toda la población debido a que su precio es cómodo y su utilidad es variada (Dane, 2012).

Por otra parte, se tiene que la participación en el PIB del sector agrícola en Colombia fue de 6,4\% (Dane 2012). A nivel nacional se cuenta con variedad de cultivos entre los que resaltan: ajonjolí, algodón, arroz, banano, cacao, café, caña de azúcar, caña panelera, cebada, cocotero, fique, flores, fríjol, frutales (uchuva, piña), hortalizas, maíz, maní, ñame, papa, palma africana, sorgo, soya, tabaco, trigo, yuca. Estos productos son utilizados en diversas industrias de acuerdo con sus propiedades y características tanto intrínsecas como extrínsecas (Rocha, 2009).

Además, "Viracachá es uno de los municipios que depende económicamente de la producción Agropecuaria; la agricultura es tradicional y de transición; el cultivo más generalizado es la papa de la variedad Parda Pastusa, Tuquerreña, y en menor porcentaje variedades para la agroindustria además de la producción de leche. Como cultivos de rotación se encuentran en su orden: Maíz, Trigo, Arveja, Haba, Avena, Cebada, Zanahoria" (Alcaldía de Viracachá- Boyacá, s.f).

Este municipio del departamento de Boyacá cuenta con diez veredas distribuidas así: Caros, Centro, Chen, Galindos, Icarina, La isla, Naranjos, Parras, Pirquatá, Pueblo Viejo y la zona urbana. Su altitud es de 2.520 metros y su población es de 3.380 habitantes. Por consiguiente, Viracachá pasa a convertirse en el escenario idóneo para generar innovación social.

Es así, como surge lo que se conoce como globalización un "proceso por medio del cual los habitantes del mundo tienen una mayor interrelación en todas las facetas de la vida: culturales, económicas, políticas, tecnológicas y del entorno", como indica Lodge (1996). Así, se tiene un proceso permanente de transformación en el cual se conjugan todos los actores del mundo. Con base en lo anterior, los mercados día a día se tornan más competitivos y cada sector en específico lucha por su supervivencia diaria enfrentándose a los cambios que se allegan con el paso del tiempo, por consiguiente el sector agrícola y de leche en el departamento de Boyacá está en la obligación de generar cambios que les permitan tornarse más competitivos no sólo allegándose a mercados nacionales sino también internacionales. 
Sin embargo, para lograr la competitividad y llegar a dichos mercados nacionales e internacionales es necesario contar con grupos de trabajo que sean liderados por aquellas personas que sobresalen por su honestidad y que son reconocidas de una u otra manera por dicho grupo. Además es importante conocer los aspectos básicos para el trabajo asociativo y la creación/gestión de organizaciones rurales y que de esta manera interactúen en escenarios propios de mercadeo.

Rodríguez y otros (2011), realizaron un estudio de proyectos de innovación social en países como Perú, Brasil, México y Colombia donde se evidencia que dichos proyectos se dan a partir de una elaboración del perfil sociocultural del sector objeto de estudio.

Cabe resaltar que el sector rural no presenta la puesta en marcha de grupos de trabajo debido a sus arraigadas costumbres y no conocen de los beneficios que estos contraen, además es importante mencionar que no se encuentran líderes en los sectores rurales puesto que su tiempo libre lo ocupan en su trabajo o no muestran interés en identificarlos, y por ultimo no se cuenta con el conocimiento teórico/práctico de la gestión de organizaciones, por lo cual es necesario plantear la pregunta o formulación del problema que se describe a continuación:

- Formulación del problema

¿Qué procesos se requieren para implementar la innovación social en los productores agrícolas y de leche del municipio de Viracachá Boyacá?

\section{ANTECEDENTES Y APROXIMACIÓN AL MARCO TEÓRICO}

Según Cárdenas (2012), la innovación social se define como un proceso de creación, imposición y difusión de nuevas prácticas sociales en distintas áreas de la sociedad. "Busca mejorar las condiciones de la sociedad como un todo; fomenta un proceso inclusivo, participativo y deliberativo con varios actores sociales; requiere liderazgo, visión y compromiso comunitario; opera exitosamente en un contexto de cooperación entre el Estado, el sector privado y la sociedad civil y, según el Centro para la Innovación Social de la Universidad de Stanford, debe ser novedosa, efectiva, eficiente, sostenible y justa para abordar un problema social" 
Por otra parte, la innovación social tiene una delimitación como concepto y un alcance para unos, según Morales, 2009, “consistiría en él un proceso a través del cual una idea se transforma en un servicio que satisface determinadas necesidades de las y los ciudadanos" o en una iniciativa original que mejora la eficacia de la acción pública. A continuación se muestra una serie de iniciativas que suelen ser reconocidas como innovaciones sociales.

Tabla 1. Iniciativas de Innovación Social

\begin{tabular}{|c|c|c|c|}
\hline $\begin{array}{c}\text { Tipo de } \\
\text { Innovación }\end{array}$ & Innovación & Ejemplos & $\begin{array}{c}\text { Características de la } \\
\text { Innovación }\end{array}$ \\
\hline Organizativa & $\begin{array}{c}\text { Instituciones que } \\
\text { aglutinan a voluntarios } \\
\text { de naturaleza } \\
\text { profesional }\end{array}$ & $\begin{array}{c}\text { Médicos sin } \\
\text { fronteras }\end{array}$ & $\begin{array}{c}\text { Vinculación de profesiones } \\
\text { y cooperación internacional }\end{array}$ \\
\hline Financiera & $\begin{array}{c}\text { Sistema de intercambio } \\
\text { alternativo al mercado }\end{array}$ & Banco de tiempo & $\begin{array}{c}\text { Sistema de trueque } \\
\text { estandarizado }\end{array}$ \\
\hline Comercial & Comercio justo & Fanco Grameen & $\begin{array}{c}\text { Pequeños créditos con } \\
\text { Kolítica de género y poca } \\
\text { burocracia }\end{array}$ \\
\hline Medioambiental & Reciclaje & The body shop & $\begin{array}{c}\text { Actividad comercial } \\
\text { con límites de abusos } \\
\text { de intermediarios y } \\
\text { beneficiando a los } \\
\text { productores de origen } \\
\text { Inserción laboral y } \\
\text { preservación del medio } \\
\text { ambiente }\end{array}$ \\
\hline Metodología & Agricultura ecológica & Sekem & $\begin{array}{c}\text { Explotación de recursos } \\
\text { endógenos y desarrollo } \\
\text { local }\end{array}$ \\
\hline & participativos & Pren works & $\begin{array}{c}\text { Porto Alegre } \\
\text { folaboración público- } \\
\text { privada para la actuación } \\
\text { local }\end{array}$ \\
\hline
\end{tabular}

Fuente: Morales (2008)

La innovación social está vinculada a la satisfacción de las necesidades humanas básicas (Moulaert y Ailenei, 2005). Esta acepción es cercana al concepto de desarrollo humano propuesto por el PNUD siguiendo la filosofía de Amartya Sen. Si se considera las necesidades humanas como lugar común desde donde surge la innovación social podríamos establecer al menos tres ejes desde los que se ejercen determinadas dinámicas o fuerzas que son: el mercado, las correspondientes a la sociedad civil y las estructuras que regulan las relaciones entre los diversos agentes implicados. 
Un eje-coordenada serían las dinámicas del mercado -oferta, demanda, eficiencia...- en el que se encuentran nuevas demandas no sólo de naturaleza puramente económica, también de índole social; en donde surgen consumidores (demandantes) con nuevas sensibilidades (no sólo quieren lo mejor o lo más barato, también quieren lo más social-ecológico, solidario...); en donde surgen iniciativas económico- sociales (empresas sociales) que aspiran a la autonomía con una finalidad económico social, etc.

Otro eje-coordenada quedaría articulado desde la óptica de la sociedad civil, en donde podemos encontrar las necesidades permanentes de las personas, en sus diversas etapas vitales (juventud, tercera edad...) y circunstancias (género, actividad laboral) en el que aparecen nuevas carencias y, por qué no, también nuevos recursos por explotar. El tercer eje quedaría articulado en el ámbito de las estructuras que regulan los marcos de actuación tanto público como privado. Es el terreno propio de la acción política para el cambio o la implantación de condiciones para que los límites de cualquier iniciativa sean configurados adecuadamente.

Teniendo en cuenta que en el proceso de innovación social se utiliza la asociatividad como un eje complementario es necesario abarcar y explicar dicho tema; a continuación se presenta la asociatividad, su definición, características, beneficios y ventajas.

Este concepto se define como "un mecanismo de cooperación empresarial en el que cada una de las empresas que participan mantiene su independencia jurídica y su autonomía gerencial, decidiendo voluntariamente su participación en un esfuerzo conjunto con los otros participantes para la búsqueda de un objetivo común.” (Rosales, 1997).

Según Fernández, M y Senior, A. (2008), conforme a la definición anteriormente mencionada, la asociatividad es un mecanismo estratégico que permite a las empresas alcanzar metas comunes casi todas ellas enfocadas al mejoramiento de la competitividad.

La asociatividad "es una estrategia colectiva, tiene carácter voluntario y no excluye a ninguna empresa por el tipo de mercado en el cual opera, es exclusiva para pequeñas y medianas empresas," (Perego, 2003).

Según Liendo y Martínez (2001), el modelo asociativo en general, sin dejar 
de lado la autonomía de las empresas participantes, posibilita la resolución de problemas comunes tales como:

- Reducción de costos.

- Incorporación de tecnología.

- Mejora del posicionamiento en los mercados.

- Acceso a mercados de mayor envergadura.

- Capacitación de recursos humanos.

- Incremento de productividad

- Acceso a recursos materiales y humanos especializados.

- Desarrollo de economías de escala.

- Disponibilidad de información.

- Captación de recursos financieros.

- Optimización de estándares de calidad.

- Desarrollo de nuevos productos.

- Ventajas competitivas, entre otros.

De otro lado, la asociatividad como estrategia de búsqueda de ventajas competitivas, se diferencia de otras estrategias orientadas al mismo fin, en el desarrollo que se otorga a la cooperación, o sea mediante el establecimiento de acuerdos con otras empresas, para la realización de una serie de actividades dentro de la cadena de valor del producto o servicio, que conduzcan a una mayor presencia de la empresa en uno o más mercados. Se considera que la asociatividad empresarial puede ser un instrumento para el desarrollo de la competitividad de las PYME en la medida que reduce los costos de transacción de las empresas y añade valor a los productos o servicios que la empresa ofrece a los consumidores, usuarios o clientes (Beltrán, 2002).

\section{METODOLOGÍA}

En el desarrollo del proyecto, se aplicaron los tipos de investigación Participación - acción y descriptiva. La participación acción teniendo en cuenta que se trabajara con las personas y se busca un acercamiento a cada uno de ellas para dar soluciones conjuntas que no se tomen desde la línea 
de mando alta sino desde la base del tejido social, que para el caso son los campesinos del sector agrícola del municipio de Viracacha, además se realizan las acciones de conocer, actuar y transformar, encaminadas a la intervención en la sensibilización de la población sobre los problemas que le rodean. La descriptiva permite describir de manera confiable la situación actual en la que se encuentran los campesinos ya que se hace uso de la observación, cuestionamiento y entrevistas que arrojan información la cual se analiza e interpreta para identificar los problemas de la comunidad.

- Población y Muestra

Según el Dane (2005) Viracachá tiene 3.380 habitantes.

Se tomó como objeto de estudio a 22 personas que hacen parte de la vereda de Chen del municipio de Viracachá, que atendieron al llamado para realizar el proceso de innovación social con productores agrícolas y lácteos.

- Estructura metodológica:

1. La población objeto de estudio son 22 personas que se interesaron en llevar a cabo el proceso de innovación social.

2. Se llevaron a cabo grupos de enfoque y entrevistas semi-estructuradas a los productores agrícolas y de leche.

3. En el análisis de la información y realización perfil socio-cultural y económico de la comunidad productora agrícola y de leche en el municipio de Viracacha - Boyacá se realizó con base en hechos reales y sobre conclusiones obtenidas, así mismo se utilizó formatos estándar de la incubadora de empresas del oriente INCUBAR BOYACÁ para obtener dicha información.

4. Para la identificación de líderes existentes en la comunidad objeto de estudio, se tiene en cuenta a aquellas personas que sobresalen por su actuar, su honestidad y a su vez reflejan transparencia ante los demás productores objeto de estudio, por otra parte se indaga sobre las personas que han tenido experiencias con trabajo asociativo.

5. En cuanto a la formación en los aspectos básicos para el trabajo asociativo y para la gestión de organizaciones asociativas rurales, a los 
productores agrícola y de leche en el municipio de Viracachá - Boyacá asociativas rurales se aplica una guía de estudios que permita dicha formación. (Guía fijada por la Incubadora de Empresas del Oriente INCUBAR BOYACÁ)

\section{Instrumentos de recolección}

- Entrevistas semi-estructuradas: Es aquella en la que, como su propio nombre indica, el entrevistador despliega una estrategia mixta, alternando preguntas estructuradas y con preguntas espontáneas. Esta forma es más completa ya que, mientras que la parte preparada permite comparar entre los diferentes candidatos, la parte libre accede profundizar en las características específicas del candidato. Por ello, permite una mayor libertad y flexibilidad en la obtención de información (Ozonas y Pérez. 2004).

- Focus Group o Grupos focales: Constituyen una técnica cualitativa de recolección de información basada en entrevistas colectivas y semi-estructuradas realizadas a grupos homogéneos. Para el desarrollo de esta técnica se instrumentan guías previamente diseñadas. Así, básicamente esta técnica consiste en reunir un número pequeño (de seis a doce personas) y con la guía de un moderador, se expresa de manera libre y espontánea sobre una temática. Ello es apropiado para la realización de este proyecto puesto que se trabajará con base al tejido social (Fontas y otros, s.f).

\section{RESULTADOS}

1. Elaborar un perfil socio-cultural y económico de la comunidad productora agrícola y de leche en el municipio de Viracachá - Boyacá.

A continuación se presenta la descripción detallada del perfil socio-cultural y económico de la población objeto de estudio de acuerdo con el desarrollo de dinámicas de grupo y variables tomadas de la caracterización (entrevista semi-estructurada) desarrollada con los formatos de la Incubadora de Empresas del Medio Oriente Incubar Boyacá. 
Figura 1. Edad

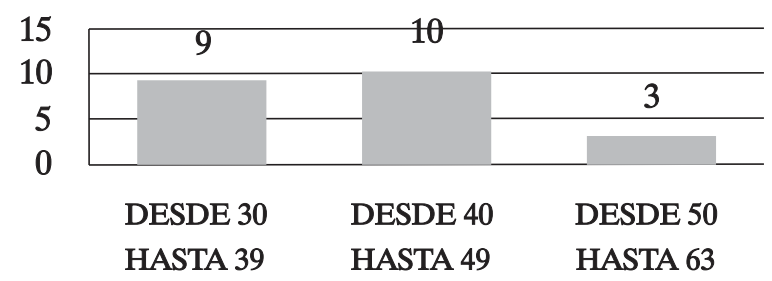

Fuente: Elaboración propia

La edad de las personas objeto de estudio se encuentra en el rango de 30 a 63 años, destacando que las personas mayores de edad se preocupan por el futuro de sus familias y también por dejar un legado para sus generaciones.

Figura 2. Género.

19

20

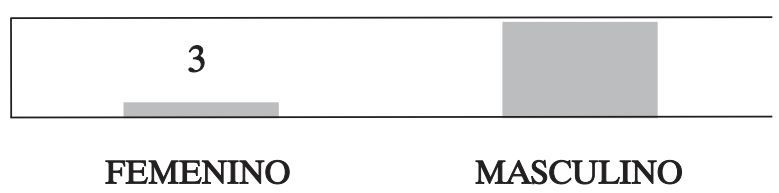

Fuente: Elaboración propia

Se evidencia que son más hombres que mujeres, los que se preocupan por ser parte del cambio social, pero no se debe desmeritar el trabajo arduo que realizan estas tres mujeres al hacer parte del proceso sin dejar de lado sus múltiples obligaciones del hogar. 
Figura 3. Nivel Educativo

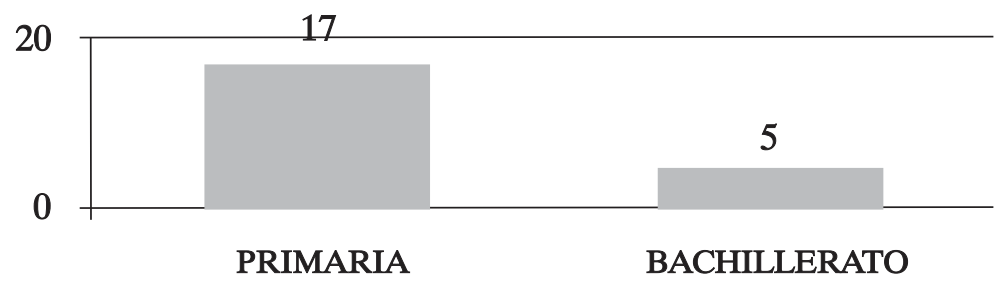

Fuente: Elaboración propia

La mayoría de personas objeto de estudio, cursaron sus estudios hasta grado segundo de primaria, algunos de ellos cursaron hasta séptimo de bachillerato y solo dos de ellos terminaron académicamente. Por otra parte, es de resaltar que a la mayoría se les dificulta el aprendizaje ya que según ellos ha transcurrido mucho tiempo desde que terminaron sus estudios; algunos de ellos no saben leer, escribir, multiplicar y dividir.

Figura 4. Estado Civil

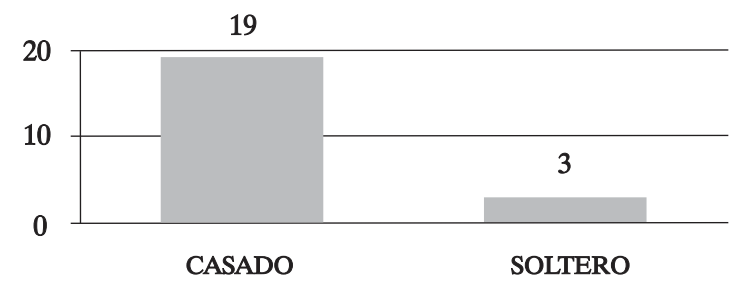

Fuente: Elaboración propia

Las personas objeto de estudio, en su mayoría son casadas. No están de acuerdo con el hecho de la separación, sin importar las condiciones en que se viva, ya que afirman haber nacido en familias con principios y valores para formar un hogar y que éste sea para siempre. 
Figura 5. Cabeza de Familia

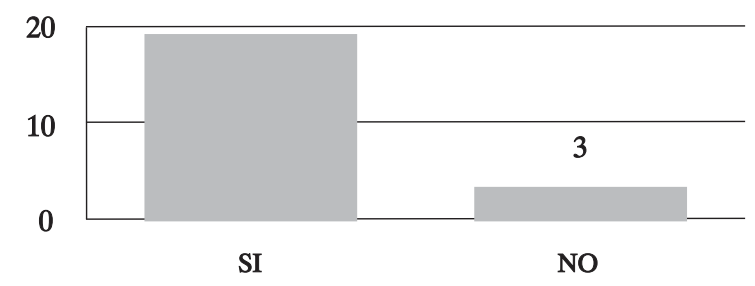

Fuente: Elaboración propia

La mayoría de personas son cabeza de familia, mantienen económicamente a los integrantes de su hogar. Hay que resaltar que estas personas cabeza de familia son los mismos hombres quienes se hacen cargo de todos los integrantes de su hogar.

Figura 6. Número de hectáreas por persona

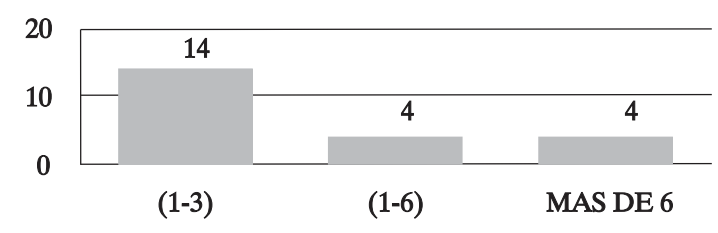

Fuente: Elaboración propia

En total, entre las personas objeto de estudio tienen 85 hectáreas, lo que muestra que poseen un potencial significativo para empezar a cultivar en conjunto, ofrecer así más productos al mercado y obtener (recibir) recursos del gobierno por el hecho de trabajar asociadamente. 
FASE PRELIMINAR EN EL PROCESO DE INNOVACIÓN SOCIAL CON LOS PRODUCTORES AGRICOLAS Y DE LECHE DEL MUNICIPIO DE VIRACACHA - BOYACÁ

Figura 7. Beneficios recibidos por parte del Gobierno

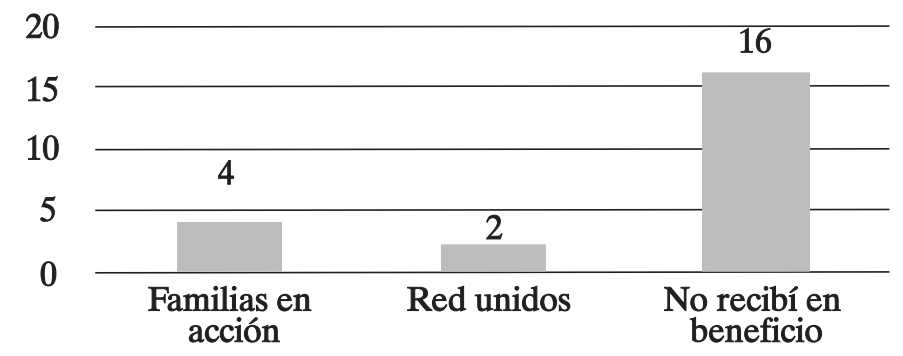

Fuente: Elaboración propia

La mayoría de personas no se acogen de beneficios por parte del gobierno, pero las personas que si lo reciben se debe a que sus esposas son quienes se han inscrito a dichos programas y han sido favorecidos.

2. Identificar liderazgos y experiencias de trabajo asociativo en la comunidad objeto de estudio.

Para la identificación de líderes existentes en la comunidad objeto de estudio se indaga sobre la caracterización de éstos y que por medio de la observación, por su actuar, su honestidad y transparencia son reconocidos por los demás integrantes de la comunidad.

Estas personas líderes se hicieron evidentes desde el momento en que se empezó el proceso de innovación social debido a que son reconocidas por el interés de surgir colectivamente y buscar beneficios para la comunidad, por medio de ellas se hacia el contacto para determinar la fecha y hora del próximo encuentro, además era el puente efectivo de comunicación entre la comunidad objeto de estudio.

En este grupo de trabajo se identifica una persona líder ya que su comunicación es efectiva, siempre busca soluciones a cada problema, confían plenamente en ella, es la cabeza visible del grupo de trabajo, posee buen carácter, motivadora, posee visión futurista, tiene facilidad para hacer reflexionar al otro.

Esta persona se encuentra en el tipo de liderazgo carismático puesto que sobresale por sus cualidades anteriormente mencionadas, se muestra 
motivadora generando satisfacción y entusiasmo dentro del grupo de trabajo, su principal error está en centrar demasiado sus esfuerzos en varias actividades puesto que al faltar puede ser causa de problema en el grupo de trabajo.

En cuanto a la experiencia asociativa (Figura 8), a partir de dinámicas de grupo se observó que algunas personas fueron parte de asociaciones y/o cooperativas pero infortunadamente estas experiencias no se reflejaron gratificantes y sembraron desconfianza en aquellas personas; sin embargo en una nueva dinámica se identificó que esta experiencia sirvió para querer seguir adelante con aquellas personas que desean subsistir y seguir con el trabajo del campo, mostrando entereza, compromiso, cumplimiento, responsabilidad, honestidad y trabajo en equipo.

Dichas personas hacen parte de la Asociación Agropecuaria de Productores Agrícolas y Lácteos de la vereda Chen - Municipio de Viracachá departamento de Boyacá; asociación que se creó poco tiempo después de iniciar el proceso de innovación social, puesto que se quiere aprovechar las ayudas y beneficios que otorga el gobierno para entidades sin ánimo de lucro que busquen mejorar las condiciones de vida de sus asociados y la comunidad en general. En la Figura 8, se observa el número de personas que han adquirido experiencias asociativas y las que aún no.

Figura 8. Experiencia asociativa

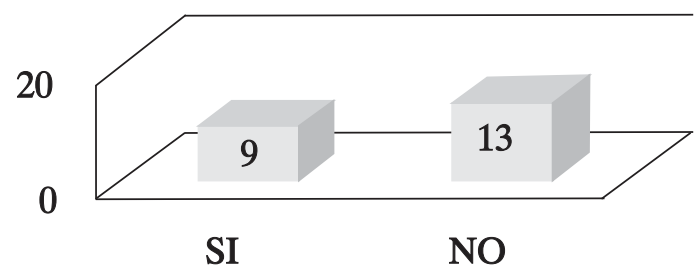

Fuente: Elaboración propia

3. Formar en los aspectos básicos para el trabajo asociativo y para la gestión de organizaciones asociativas rurales, a los productores agrícolas y de leche en el municipio de Viracachá - Boyacá. 
En primera estancia, dicho proceso se realizó en alianza con la Incubadora de Empresa del Oriente Incubar Boyacá, ya que ellos están adelantando un programa de fortalecimiento asociativo, empresarial y en gestión de TIC's a pequeños productores agrícolas que busca mejorar las condiciones de vida de las personas.

En la realización de esta alianza se otorgaron tareas a realizar por las diferentes partes; la incubadora de empresas por su parte hizo entrega de los formatos que se debían implementar; a su vez entregó el plan de acción a llevar a cabo del cual se obtuvo:

- Desarrollo de una dinámica de grupo donde se realizó un diagnóstico previo a la implementación del proceso de innovación social con el fin de identificar los aspectos en los cuales la comunidad objeto de estudio tenía desconocimiento y era de vital importancia para el trabajo asociativo, por lo cual se realizó el siguiente análisis DOFA:

\section{Tabla 2. Análisis DOFA}

\begin{tabular}{|c|c|c|}
\hline $\begin{array}{c}\text { ANALISIS } \\
\text { DOFA }\end{array}$ & $\begin{array}{l}\text { OPORTUNIDADES } \\
\text { 1. Hay entidades gubernamenta- } \\
\text { les que desean ayudar a los pe- } \\
\text { queños agricultores para la sub- } \\
\text { sistencia de estos. } \\
\text { 2. Recursos económicos para pe- } \\
\text { queños agricultores que busquen } \\
\text { mejorar las condiciones de vida } \\
\text { de la comunidad en general. }\end{array}$ & $\begin{array}{l}\text { AMENAZAS } \\
\text { 1. Los recursos que otorga el go- } \\
\text { bierno son para entidades sin ánimo } \\
\text { de lucro. } \\
\text { 2. No cuentan con los recursos ne- } \\
\text { cesarios para la creación de una or- } \\
\text { ganización sin ánimo de lucro. }\end{array}$ \\
\hline $\begin{array}{l}\text { FORTALEZAS } \\
\text { 1. Disposición para ge- } \\
\text { nerar cambio positivo } \\
\text { en la comunidad. } \\
\text { 2. Manejan un nivel } \\
\text { alto de comunicación } \\
\text { entre las personas ob- } \\
\text { jeto de estudio. } \\
\text { 3. Se presenta asig- } \\
\text { nación de autoridad, } \\
\text { liderazgo y responsabi- } \\
\text { lidades. }\end{array}$ & $\begin{array}{l}\text { F+O } \\
\text { Identificar a la persona líder que } \\
\text { será también la representante le- } \\
\text { gal y distribuir funciones a cada } \\
\text { asociado de tal manera que se } \\
\text { haga uso de las ayudas guberna- } \\
\text { mentales y contribuir a mejorar las } \\
\text { condiciones de vida y sociales de } \\
\text { la comunidad involucrada. }\end{array}$ & $\begin{array}{l}\text { F+A } \\
\text {-Crear una organización sin ánimo } \\
\text { de lucro que busque mejorar las } \\
\text { condiciones de vida de sus asocia- } \\
\text { dos. } \\
\text {-Asignar a un grupo de personas } \\
\text { para que sean quienes lideren los } \\
\text { procesos de búsqueda de recursos, } \\
\text { una vez creada la asociación. }\end{array}$ \\
\hline
\end{tabular}




\begin{tabular}{|l|l|l|}
\hline \multicolumn{1}{|c|}{ DEBILIDADES } & \multicolumn{1}{|c|}{ D+O } & \multicolumn{1}{c|}{ D+ A } \\
$\begin{array}{l}\text { 1. No poseen conoci- } \\
\text { miento sobre la crea- } \\
\text { ción y manejo de una } \\
\text { entidad sin ánimo de } \\
\text { lucro. }\end{array}$ & $\begin{array}{l}\text { Buscar ayuda en aquellas enti- } \\
\text { dades gubernamentales para la } \\
\text { creación de una asociación y que } \\
\text { a su vez fortalezcan a la entidad } \\
\text { con capacitaciones en temas rela- } \\
\text { cionados con la creación y fortale- } \\
\text { cimiento de esta. }\end{array}$ & $\begin{array}{l}\text { Aprovechar al máximo las capaci- } \\
\text { taciones que brindan las entidades } \\
\text { de apoyo. }\end{array}$ \\
$\begin{array}{l}\text { 2. Baja concentración } \\
\text { académica de las per- } \\
\text { sonas. }\end{array}$ & & \\
\hline
\end{tabular}

Fuente: Elaboración Propia

Una vez realizado el análisis DOFA se obtuvieron las siguientes estrategias: $\mathrm{F}+\mathrm{A}$

- Crear una organización sin ánimo de lucro que busque mejorar las condiciones de vida de sus asociados

- Asignar a un grupo de personas para que sean quienes lideren los procesos de búsqueda de recursos.

$\mathrm{F}+\mathrm{O}$

- Identificar a la persona líder que será también la representante legal y distribuir funciones a cada asociado de tal manera que se haga uso de las ayudas gubernamentales y contribuir a mejorar las condiciones de vida y sociales de la comunidad involucrada.

$\mathrm{D}+\mathrm{O}$

- Buscar ayuda en aquellas entidades gubernamentales para la creación de una asociación y que a su vez fortalezcan a la entidad con capacitaciones en temas relacionados con la creación y fortalecimiento de esta.

$\mathrm{D}+\mathrm{A}$

- Aprovechar al máximo las capacitaciones que brindan las entidades de apoyo.

Cabe resaltar, que el desarrollo de estas estrategias se realizaron en conjunto con las personas objeto de estudio, quienes colaboraron al máximo para el total cumplimiento de las actividades y la obtención de buenos resultados.

Se vió la necesidad de desarrollar capacitaciones para lograr un impacto 
social en la comunidad y fortalecer las habilidades y destrezas con las que contaba el grupo objeto de estudio, para este proceso se indagó sobre las diferentes temáticas que se debía abordar en dichas capacitaciones y que fueran de fácil aprendizaje ya que el rango de edad en el cual se encuentran le dificulta el entendimiento de algunos aspectos.

Se obtuvieron tres impactos como resultado, que son:

1. Social: a través de sesiones de grupo que lograron identificar liderazgos, experiencias personales, laborales, buscar nuevas formar de subsistir con su actividad económica sin dejar de lado el trabajo en grupo, identificar habilidades y destrezas de cada integrante y crear un ambiente de confianza y delegación de tareas con responsabilidad y compromiso entre los actores.

2. Económico: puesto que se logró la creación de un modelo de negocio que acoge a cada uno de las personas objeto de estudio y de esta manera buscan en conjunto un objetivo principal que es el de además de dejar un legado a sus generaciones, generar valor.

3. Ambiental: ya que en este momento están implementando las buenas prácticas agrícolas que ayudan a ser cada vez más competitivos y así poder abarcar mercados nacionales e internacionales. Esta decisión se tomó desde la base del tejido social observando que esta implementación contrae muchos beneficios y fue de común acuerdo, mostrando siempre ayudar a su grupo y mejorar las condiciones de vida de todos los integrantes de éste.

\section{CONCLUSIONES}

1. Las personas dedicadas a la producción agrícola y láctea presentan bajo nivel educativo, lo que implica que no están en condiciones de mantener un cargo administrativo, por lo que necesita de mayor atención y capacitación en temas relacionados.

2. El desarrollo de la innovación social se ubicó en una zona rural con niveles de pobreza relativamente altos con relación al promedio nacional y tamaño de predio, según el Instituto Geográfico Agustín Codazzi (2012).

3. En el desarrollo del proceso de innovación social, el papel del gobierno departamental fue evidente ya que cuentan con presupuesto designado para realizar programas de fomento y fortalecimiento a organizaciones solidarias. 
4. Según Rodríguez y otros (2011), en el análisis realizado a informes de casos de innovación social en Argentina, Brasil, Colombia, Ecuador, Haití, México y Perú, afirman que las personas a quienes se les aplica el proceso no muestran resistencia al cambio, por el contrario escudriñan soluciones a sus problemas y a su vez buscan apoyo económico de los gobiernos locales; afirmación que también se presenta en los productores agrícolas y de lácteos del municipio de Viracachá.

5. El impacto que generó la implementación del proceso de innovación social se ve reflejado en la creación de oportunidades, desarrollo del saber y del hacer de las personas objeto de estudio, la generación de bienestar de la comunidad en general y la toma de decisiones por parte de la organización.

\section{RECOMENDACIONES}

Como institución de conocimiento realizar mayor número de proyectos implementando alianzas estratégicas con entidades gubernamentales que busquen la mejora continua de la región.

También es importante los proyectos encaminados a la construcción de trabajo asociativo ya que los campesinos no cuentan con un nivel alto de educación y sería de gran importancia empezar por enseñar a aquellas personas a defenderse en actividades como lo son leer, escribir, sumar, restar, dividir, y utilizar la tecnología como una herramienta de comercialización. Además capacitar en temas relacionados con el manejo tributario, financiero, presupuestal y administrativo de organizaciones. 
FASE PRELIMINAR EN EL PROCESO DE INNOVACIÓN SOCIAL CON LOS PRODUCTORES AGRICOLAS Y DE LECHE DEL MUNICIPIO DE VIRACACHA - BOYACÁ

\section{REFERENCIAS BIBLIOGRAFICAS}

Alcaldía de Viracacha- Boyacá. (s.f). Recuperado 25/02/14 de: http:// viracacha-boyaca.gov.co/presentacion.shtml.

Beltrán, E. (2002). Desafíos y oportunidades de las PYMES construyendo una agenda de desarrollo. Madrid: Fundes. pp. 110 - 146.

Cárdenas, R. (2012). El camino hacia la innovación social. Colombia: Bogotá.

Cepal. (2011). Innovación social y sus delimitaciones. Recuperado 15/11/13 de www.eclac.org/id.asp?id=43497

Dane (2005). Censo. Recuperado 28/03/14 de: http://www.dane.gov.co/ index.php/poblacion-y-demografia/censos.

Dane (2012). Producto Interno Bruto - Cuatro Trimestre y Total Anual 2012. Fernández, M y Senior, A. (2008). El desarrollo local sobre la base de la asociatividad empresaria: una propuesta estratégica. Opción, año 24, No. 57. p. 77.

Fontas, C., Conçalves, F., Vitale, M y Viglietta, D. (2010). La técnica de los grupos focales en el marco de la investigación socio- cualitativa. Argentina: Buenos Aires

Instituto Geográfico Agustín Codazzi. (2012). Atlas de la Distribución de la Propiedad Rural en Colombia. pp. 62-70.

Liendo, M. y Martínez, A. (2001). Asociatividad: una alternativa para el desarrollo y crecimiento de las pymes. Sextas Jornadas "Investigaciones en la Facultad" de Ciencias Económicas y Estadística.

Lodge G, (1996). Administrando la globalización en la era de la independencia. Editorial Panorama S., México.

Moulaert, F. y Ailenei, O. (2005): La economía social, tercer Sector y Relaciones Solidaridad: Síntesis conceptual de la historia al presente. Estudios Urbanos, Vol. 42, No 11 , pp. 2037-2053.

Morales, A. (2008). Innovación social: un proceso emergente en las dinámicas de desarrollo. Revista de Fomento Social, Nº 63, pp.411444. 
Morales, A. (2009). Innovación social: un ámbito de interés para los servicios sociales. España.

Ozonas, L y Pérez, A. (2004). La entrevista semiestructurada. Notas sobre una práctica metodológica desde una perspectiva de género. Facultad de Humanidades.

Perego, L. (2003). Competitividad y Clúster Productivos. Editor: Juan Carlos Martínez. p. 17-18. Argentina.

Revista Semana, (2013). Paro Agrario: las dos caras de la protesta. Recuperado el 29/12/2013 de http://www.semana.com/nacion/ articulo/paro-agrario-las-dos-caras-de-la-protesta/356110-3.

Rocha, P. (2009). Situación y Perspectivas de los cultivos transgénicos en Colombia. Bogotá: IICA. pp. 1-23

Rodríguez, L., Bernal, M. y Cuervo, L. (2011). Innovación social y desarrollo económico social. Santiago: CEPAL

Rodríguez, L., Bernal, M. y Cuervo, L. (2011). Innovación social y desarrollo económico local. Serie políticas sociales $n^{\circ} 170$. Santiago: CEPAL pp. 1-84

Rosales (1997). Citado por NARVAEZ, Mercy; FERNANDEZ, Gladys y SENIOR,Alexa.El desarrollo local sobre la base de la asociatividad empresaria: una propuesta estratégica. En: Opción, año 24, No. 57. 2008. p. 77. ISSN: 1012-1587 